\title{
Abordaje de la complejidad psicosocial en pacientes con cáncer
}

\author{
Francisco Luis Gil Moncayo $^{\mathrm{a}, \mathrm{b}, *}$, Jorge Maté Méndez ${ }^{\mathrm{a}, \mathrm{b}}$, Cristian Ochoa Arnedo ${ }^{\mathrm{a}}$, Anna Casellas-Grau ${ }^{\mathrm{a}}$, \\ Jordi Trelis Navarro ${ }^{c}$ y Josep Maria Borràs Andrés ${ }^{d}$

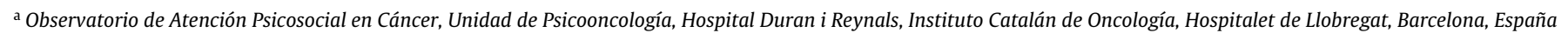 \\ b Grupo de Investigación en Estrés y Salud (GIES), Facultad de Psicología, Universidad Autónoma de Barcelona, Bellaterra, Barcelona, España \\ ${ }^{c}$ Dirección Asistencial, Hospital Duran i Reynals, Instituto Catalán de Oncología, Hospitalet de Llobregat, Barcelona, España \\ d Plan Director de Oncología de Cataluña, Hospital Duran i Reynals, Instituto Catalán de Oncología, Hospitalet de Llobregat, Barcelona, España
}

\section{INFORMACIÓN DEL ARTÍCULO}

\section{Historia del artículo:}

Recibido el 4 de diciembre de 2018

Aceptado el 7 de marzo de 2019

On-line el xxx

\section{Palabras clave:}

Cáncer

Oncología

Psicooncología

Psicosocial

Complejidad

\section{R E S U M E N}

Antecedentes y objetivo: Evaluar el malestar emocional y el nivel de complejidad de pacientes presentados al Comité de Atención Psicosocial.

Materiales y métodos: Estudio pre-post con un solo grupo en pacientes con cáncer. De los 144 pacientes, 27 fueron derivados por el comité a especialistas del área psicosocial, y sus niveles de malestar emocional y de complejidad fueron revisados un mes después.

Resultados: Tras ser atendidos según indicaciones del comité, los pacientes mostraron un descenso significativo de los valores en malestar emocional: la media inicial de 8 puntos en la escala visual analógica de malestar emocional descendía a 5,8 puntos. El descenso se reflejó también en los niveles de complejidad: antes de ser revisados, el 70,4\% de los pacientes mostraban un alto nivel de complejidad y el 7,4\%, un nivel bajo. Después de ser atendidos, los porcentajes de pacientes con alta complejidad se redujeron al $48,1 \%$ en los enfermos con alta complejidad, y aumentaron al 22,3\% en los de baja complejidad.

Conclusiones: El comité ofrece un instrumento para derivar los casos de mayor complejidad que requieren atención preferente y multidisciplinar, permitiendo optimizar recursos, por su eficacia en la resolución de casos complejos.

(c) 2019 Elsevier España, S.L.U. Todos los derechos reservados.

\section{Approaching psychosocial complexity in patients with cancer}

\begin{abstract}
A B S T R A C T
Background and aim: To assess emotional distress and complexity of patients referred to the Psychosocial Committee.

Material and methods: A pre-post single group study was performed in a sample of oncological patients. From the 144 patients referred to the committee, 27 were attended by psychosocial specialists. The patients' levels of emotional distress and psychosocial complexity were reviewed one month later. Results: After having been attended according to the committee's indications, the patients showed significant decreases in emotional distress. The initial mean of 8 points on the emotional distress scale decreased to 5.81 points after having been referred to the specialist. This decline was also observed in psychosocial complexity. Before attendance, $70.4 \%$ patients showed high levels of complexity, and $7.4 \%$ showed low levels. After attendance, the percentages of patients with high levels of psychosocial complexity reduced to $48.1 \%$ and patients with low complexity increased to $22.2 \%$.

Conclusions: The committee provides an instrument to refer patients who show high levels of psychosocial complexity and require preferential and multidisciplinary attention. The committee optimizes resources due to its efficiency in resolving complex cases.
\end{abstract}

(C) 2019 Elsevier España, S.L.U. All rights reserved.

* Autor para correspondencia.

Correo electrónico: fgil@iconcologia.net (F.L. Gil Moncayo). 


\section{Introducción}

Ser diagnosticado de cáncer genera un impacto psicológico que puede derivar en niveles significativos de malestar emocional ${ }^{1,2}$. Aunque estos síntomas pueden remitir espontáneamente, en cerca de una cuarta parte de los pacientes puede resultar en trastorno mental ${ }^{3-5}$, especialmente en trastorno adaptativo, presente en el $70-77 \%$ de los pacientes ${ }^{3,4}$. En estos casos, si no se ofrece una priorización en la atención psicosocial, los síntomas pueden perdurar en el tiempo, dado que un tercio de los pacientes oncológicos muestran malestar emocional a largo plazo ${ }^{6}$. La atención psicosocial en cáncer forma parte de todo modelo integral para reducir el impacto vital de la enfermedad. Las intervenciones psicosociales han probado su eficacia en la ayuda a pacientes y familiares para afrontar las situaciones complejas y desbordantes derivadas del diagnóstico, así como económicamente eficientes en el análisis de coste-efecto ${ }^{7}$. La adecuada atención psicooncológica recomendada por la Guía Europea para la Calidad Nacional de Programas de Control en Cáncer (European Guide for Quality National Cancer Control Programmes) $)^{8,9}$ se compone de los siguientes puntos: 1) formación de los profesionales sobre aspectos psicosociales en cáncer; 2) inclusión de la valoración del nivel de malestar emocional como sexto signo vital en pacientes con cáncer; 3) utilización de tratamientos basados en la evidencia para el manejo de síntomas y necesidades psicosociales; 4) desarrollo de criterios de práctica clínica en los servicios de psicooncología; 5)implementación e integración de programas de atención psicooncológica dentro de los equipos de atención multidisciplinar, y 6) compromiso de los proveedores de atención oncológica, públicos o privados, de incluir la atención psicosocial para una adecuada atención integral al paciente y a la familia.

Según la Declaración Institucional para el Desarrollo de la Atención Multidisciplinar en Cáncer en España, y durante la Presidencia Portuguesa de la Unión Europea ${ }^{10}$, los equipos de atención multidisciplinar han sido identificados como el mejor abordaje para la atención en cáncer, contribuyendo al trabajo coordinado de todos los profesionales envueltos en el diagnóstico y el tratamiento del paciente $\mathrm{e}^{11-13}$. Así, algunos países ya han creado estándares de atención psicosocial oncológica ${ }^{14,15}$. El Hospital Duran i Reynals, del Instituto Catalán de Oncología (ICO-DiR), fue pionero en este ámbito en España, disponiendo de un Comité de Atención Psicosocial (CPS) desde el año 2002 y elaborando los principios de una guía de atención psicosocial que se encuentran detallados en el modelo de atención psicosocial en oncología (estándares) ${ }^{16}$. Este CPS se creó con el objetivo de mejorar la atención al enfermo oncológico y a sus familiares, favoreciendo la coordinación de las distintas disciplinas implicadas y creando circuitos asistenciales, sanitarios y comunitarios rápidos dentro del ámbito psicosocial.

En la línea de las recomendaciones anteriormente expuestas, proponemos este estudio para determinar el beneficio de un modelo de gestión de atención psicosocial basado en un CPS como un nuevo modelo organizativo para la gestión de pacientes complejos -que requieren atención multidisciplinar e interdisciplinarque ayuda a priorizar sus necesidades de atención psicosocial, a mejorar su bienestar emocional en el menor tiempo posible y a optimizar los recursos asistenciales de que debe disponer todo centro sanitario que provee de atención médica al paciente con cáncer. El CPS es un instrumento de apoyo a todo profesional implicado en tareas asistenciales (enfermería y medicina) en relación con la derivación de los pacientes y familiares a los recursos de atención psicosocial que precisen. A su vez, tiene unas funciones y ámbitos de actuación específicos, cuyos principales objetivos se pueden resumir en la valoración, la derivación y la atención continuada de la complejidad psicosocial del paciente y la familia, tanto a nivel ambulatorio como de ingreso hospitalario (fig. 1).

\section{Material y métodos}

\section{Participantes}

Los pacientes ambulatorios oncológicos son visitados en primer lugar por el equipo médico y enfermería. Los casos que, según el criterio del profesional, muestran algún signo de complejidad psicosocial son derivados al comité mediante la entrega del acta del CPS (fig. 2) a la administrativa, para considerar su prioridad de atención psicosocial. Entre los meses de enero y junio de 2018, 144 casos fueron entregados y discutidos en el comité. De estos, 27 fueron priorizados y evaluados antes y después de la atención indicada por el comité.

\section{Instrumentos de medida}

Listado de indicadores de complejidad psicosocial. En el acta del CPS (fig. 2) se incluyen los ítems acordados por los miembros del CPS como indicadores de complejidad psicosocial. Para facilitar su identificación, se propuso una tabla resumen consensuada de indicadores de vulnerabilidad y riesgo psicosocial (fig. 3). Los indicadores de complejidad psicosocial incluyen parámetros sociofamiliares, psicológicos y personales que permiten la clasificación de la situación del paciente en tres niveles: baja, media y alta complejidad psicosocial. A partir de julio de 2018, los criterios de complejidad psicosocial que se utilizan se sustentan en los resultados de un estudio Delphi, pendiente de publicación, validado por expertos nacionales e internacionales. Los criterios de alta complejidad en cáncer consensuados mediante la discusión entre expertos de acuerdo a la metodología Delphi son los siguientes: a) Área clínica-física: dificultades en el control de síntomas. b) Área sociofamiliar: ausencia del cuidador; paciente con miembros vulnerables a su cargo. c) Área psicoemocional: ansiedad severa; depresión severa; ideación autolítica; desesperanza. d) Área espiritual: pérdida del sentido y del propósito de vida.

Escala de malestar emocional. Se encuentra dentro del acta del CPS y evalúa el nivel de malestar emocional en pacientes con cáncer mediante una escala visual numérica de 0 a 10 , lo que permite a los profesionales sanitarios llevar a cabo una atención específica (véase fig. 1).

\section{Procedimiento}

\section{Principios básicos de la práctica psicosocial en oncología}

El CPS se basa en los principios básicos de la práctica psicosocial en oncología, e incluye: 1) la atención psicosocial en oncología forma parte del cuidado integral de las personas enfermas de cáncer y su familia; 2) el enfermo y la familia es la unidad básica de atención psicosocial; 3 ) los pacientes y familiares deben ser informados y tener acceso a los servicios de atención psicosocial de la institución; 4) los profesionales deben respetar la dignidad y la integridad del individuo; 5) la atención psicosocial debe estar disponible a lo largo de todas las fases de la enfermedad oncológica; 6) los aspectos psicológicos y sociales deben ser valorados por el equipo asistencial a través de indicadores de riesgo-vulnerabilidad y/o necesidades psicosociales validados, los cuales serán acordados y aprobados por el CPS; 7) los miembros de los equipos profesionales interdisciplinarios deben respetar la experiencia y el conocimiento de cada uno de ellos; 8) la práctica psicosocial debe estar basada en la evidencia y sujeta a posibles cambios para aumentar su efectividad, y 9) la formación continuada y la investigación por parte de los miembros del equipo psicosocial son esenciales para la práctica asistencial.

\section{Composición del CPS}

El CPS está formado por al menos un profesional de cada una de las siguientes áreas: psicooncología, psiquiatría, enfermería clínica 


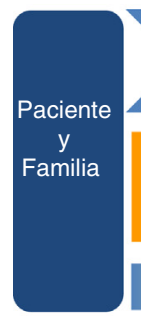

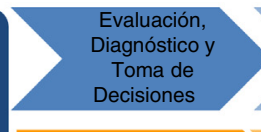

Atención Ambulatoria
Seguimiento y

Atención

Continuada

\section{Atención} Hospitalaria
Coordinación y

Trabajo en Red

Comunidad
Formación e Investigación

\section{ÁMBITOS DE ACTUACIÓN}

Figura 1. Funciones y ámbitos de actuación del Comité Psicosocial en Oncología (CPS).

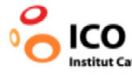

ICO

\section{ACTA DEL COMITÉ PSICOSOCIAL (CPS)}

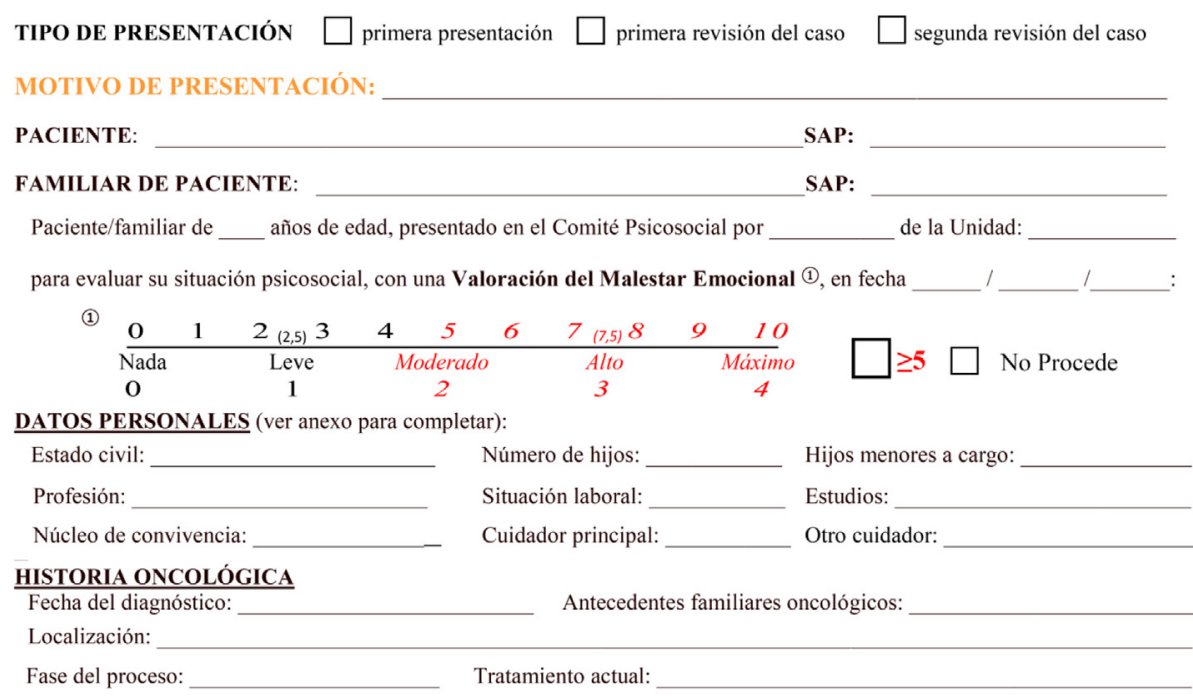

\section{CRITERIOS DE PRESENTACIÓN POR COMPLEJIDAD (ver anexo para ampliar)}

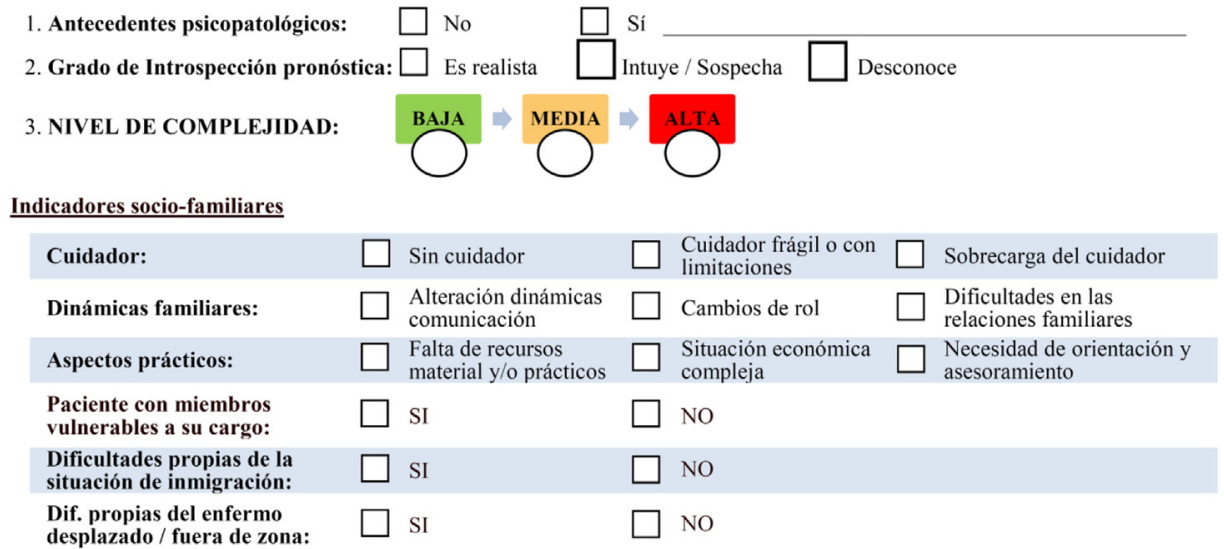

VALORACIÓN DEL COMITÉ PSICOSOCIAL (responsables):

Derivación PSO: Derivación PSQ: Derivación TSO Derivación Otros: Revisión:

Figura 2. Acta del Comité Psicosocial (CPS).

y trabajo social. Asimismo, se pueden incorporar dentro del comité profesionales de otros equipos médicos (oncólogo, hematólogo, paliatólogo), según las características de los pacientes discutidos. Todos estos profesionales disponen de formación adecuada sobre los aspectos psicosociales en cáncer, reuniendo las siguientes cualificaciones: 1) diplomados/as en trabajo social, licenciados/as en enfermería clínica (gestoras de casos de las unidades funcionales de cáncer), licenciados en psicología, licenciados en medicina y/o 


\begin{tabular}{|c|c|}
\hline ALTA & TSO \\
\hline $\begin{array}{l}\text { Antecedentes i/o psicopatología actual descompensantes. } \\
\text { Abuso substancias tóxicas. } \\
\text { Problemas de adherencia terapéutica. } \\
\text { Ideación / intento de autolisis. } \\
\text { Problemas de comunicación / información. } \\
\text { Dificultad en el control de síntomas. }\end{array}$ & $\begin{array}{l}\text { Paciente sin cuidador identificado. } \\
\text { Cuidador frágil o con limitaciones. } \\
\text { Paciente con miembros vulnerables a su cargo. } \\
\text { Situación económica compleja. } \\
\text { Necesidad de recursos materiales y/o prácticos. }\end{array}$ \\
\hline \multicolumn{2}{|c|}{ MEDIA } \\
\hline $\begin{array}{l}\text { Tratamientos oncológicos de alta complejidad. } \\
\text { Problemas de comunicación en pacientes con hijos menores a } \\
\text { su cargo. } \\
\text { Hijos adolescentes y adultos jóvenes < } 30 \text { años. } \\
\text { Afectación importante de la imagen corporal. } \\
\text { Dificultad de adaptación al diagnóstico, recidiva y enf. avanzada. } \\
\text { Preocupación existencial / espiritual. } \\
\text { Malestar emocional persistente. }\end{array}$ & $\begin{array}{l}\text { Sobrecarga del cuidador. } \\
\text { Alteración en las dinámicas de comunicación familiares. } \\
\text { Dificultades propias de la situación de inmigración. } \\
\text { Dificultades propias de la situación de enfermo } \\
\text { desplazado. }\end{array}$ \\
\hline \multicolumn{2}{|c|}{ BAJA } \\
\hline $\begin{array}{l}\text { Problemas psicológicos del familiar / paciente asociados } \\
\text { enfermedad. } \\
\text { Pacientes en proceso de duelo. } \\
\text { Eventos estresantes. } \\
\text { Bajo apoyo social. } \\
\text { Cirugía de reducción de riego en el ámbito del consejo genético. } \\
\text { Paciente concarga familiar importante. } \\
\text { Disfunción sexual. } \\
\text { Alteraciones cognitivas (memoria, concentración y/o atención). }\end{array}$ & $\begin{array}{l}\text { Dinámicas familiares con cambios de rol. } \\
\text { Dificultades en las relaciones familiares. } \\
\text { Necesidad de orientación y asesoramiento. } \\
\text { Paciente y familia con malestar emocional que } \\
\text { invalida. }\end{array}$ \\
\hline
\end{tabular}

Figura 3. Nivel de complejidad: indicadores de vulnerabilidad y riesgo psicosocial.

especialistas en psiquiatría, y 2) la psicooncología es considerada como un área de la psicología enfocada a los aspectos psicosociales en cáncer, por lo que la formación del psicooncólogo requiere una supervisión reglada, de acuerdo a unos mínimos de contenido teórico.

\section{Funciones de los componentes del CPS}

Responsable: 1) coordinación de la presentación y discusión de los casos presentados al CPS; 2) establecimiento de contactos y coordinación con los profesionales a cargo del paciente y con otros recursos psicosociales dentro del ámbito sanitario externo y de la comunidad.

Psicooncólogo: 1) evaluación, diagnóstico y tratamiento psicológicos a pacientes con cáncer y familiares presentados al CPS; 2) validación y orientación a enfermería clínica en la detección de casos clínicos, o de alta complejidad psicosocial, que necesitan ser valorados dentro del CPS, y 3) asesoramiento y validación a las enfermeras clínicas en la gestión de emociones del paciente o de la familia.

Psiquiatra: consultor, diagnóstico y tratamiento psicofarmacológico en salud mental en el ámbito oncológico.

Trabajador social: 1)detección de situaciones sociofamiliares difíciles en relación con el diagnóstico y el proceso de tratamiento; 2)identificación de los elementos necesarios del enfermo y/o de la familia para poder hacer frente a su situación y elaborar un plan de atención individualizado; 3 ) establecimiento de un plan de trabajo donde aparezcan los recursos socioeconómicos de los pacientes y familiares de los que se puedan beneficiar, estableciendo un vínculo y una correcta derivación; 4) realización de intervenciones que reduzcan el impacto sociofamiliar del enfermo con cáncer.

Enfermería clínica: 1) detección y presentación de casos dentro del CPS que ofrezcan criterios clínicos o de alta complejidad que requieran una atención psicosocial priorizada; 2 ) información a los miembros del equipo médico de referencia de la decisión tomada dentro del CPS sobre el caso presentado; 3) asesoramiento a sus equipos médicos o unidades funcionales de referencia sobre la evaluación y el manejo psicosocial de enfermos oncológicos y sobre el funcionamiento del CPS, y 4) intervenciones psicoeducativas o counselling a pacientes y familiares.

Equipo médico (oncología, consejo genético, hematología y/o cuidados paliativos): 1)presentación de casos al comité; 2) asesoramiento a los miembros del comité sobre manejo médico y de control de síntomas; 3 ) toma de decisiones sobre la derivación del caso a otros equipos médicos.

Equipo administrativo: 1) recogida y presentación de casos en el CPS; 2)cumplimentación e introducción del acta de los casos presentados en la historia clínica del paciente; 3) programación de visitas una vez el responsable ha revisado y establecido el orden de priorización de los casos presentados al CPS.

Consultor: 1) revisión de la organización del CPS; 2 ) introducción de mejoras dentro del CPS; 3) organización y supervisión de la formación continuada de los miembros.

\section{Criterios de derivación y niveles de complejidad}

Tal y como se ha descrito anteriormente, los niveles de complejidad utilizados por el CPS desde el año 2017 hasta julio de 2018 han sido el de alta, media y baja complejidad, según criterio del profesional que presentaba el caso. Los resultados del presente estudio hacen referencia a esta clasificación. Desde julio de 2018, los casos derivados al comité son valorados como de alta o no alta complejidad. Tras el estudio Delphi anteriormente mencionado, realizado durante 2018 desde el Instituto Catalán de Oncología, y de acuerdo con el consenso final de expertos sobre los criterios de complejidad psicosocial en cáncer, los pacientes que presentan altos niveles de complejidad psicosocial deben ser priorizados y visitados en menos de dos semanas desde la fecha que se presentaron en el comité. Estos casos serán revisados dentro del CPS y, a criterio del profesional, pueden ser presentados de nuevo un mes después, con el objetivo de conocer los cambios logrados a nivel de malestar emocional y nivel de complejidad, tras haber sido visitados por alguno de los miembros del CPS. 


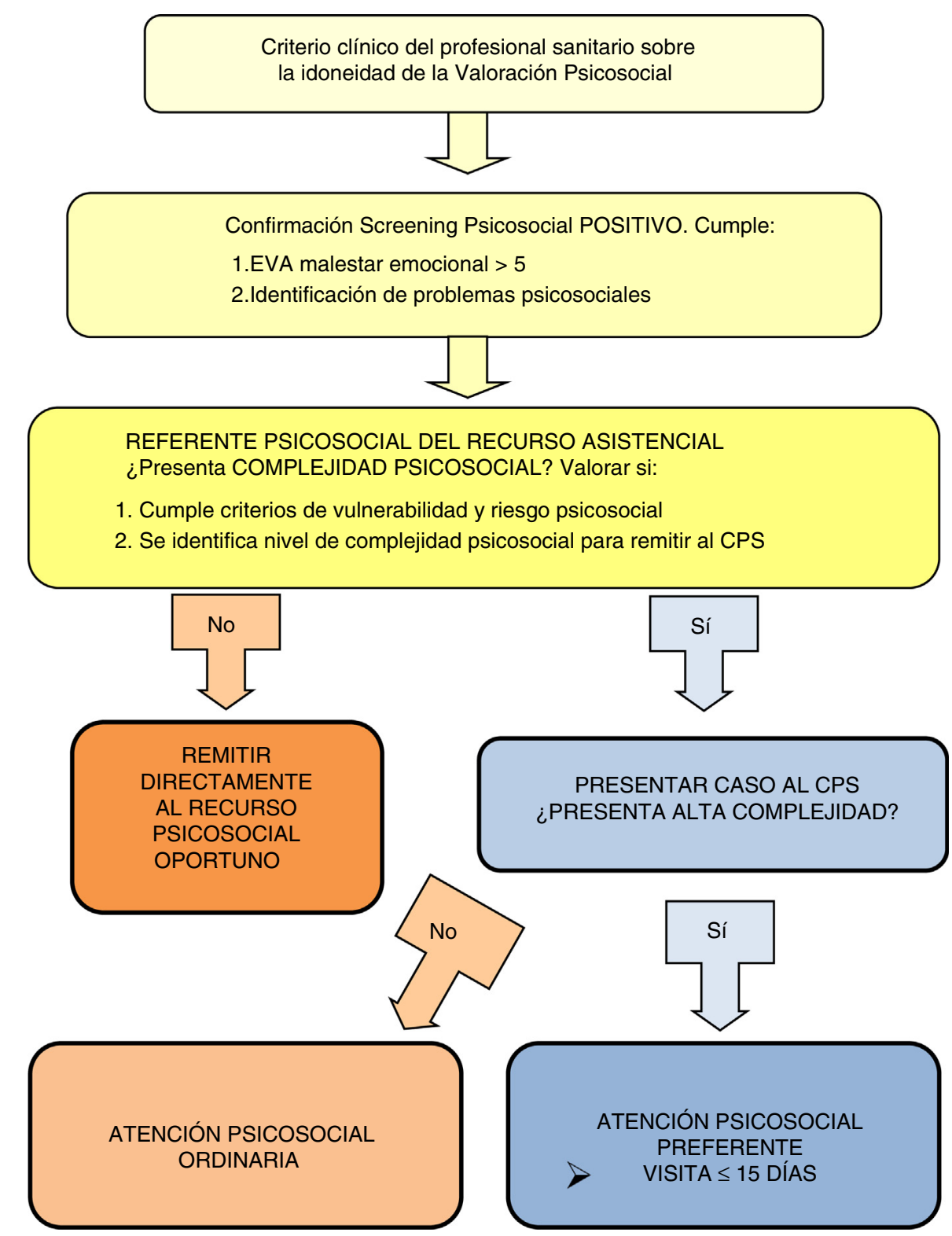

Figura 4. Circuito de evaluación y derivación de casos al CPS por complejidad psicosocial.

\section{Organización del CPS}

La misión del CPS es agrupar a diferentes profesionales implicados en la asistencia psicosocial en cáncer en un espacio físico común para ofrecer una atención integral, coordinada, completa y de calidad al paciente o familiar. No es solo una reunión entre profesionales, sino una reunión que posibilita estar en contacto, solucionar problemas psicosociales y optimizar recursos asistenciales, priorizando y agilizando los procesos de asistencia.

Los pacientes o familiares serán derivados al CPS por parte de sus miembros, así como por otros profesionales no miembros del CPS, que pueden asistir en la reunión para presentar casos. Los profesionales dispondrán de indicadores de derivación al comité según el grado de complejidad y otros elementos psicosociales y médicos recogidos en el acta del CPS (fig. 2 ), que podrán enviar rellenada a la administrativa/o, antes de la reunión. Todos los profesionales de la institución podrán consultar con el miembro de su equipo referente del CPS para valorar la idoneidad de presentar un caso. De manera esquemática, en las figuras 4 y 5 se exponen los pasos a seguir para presentar un caso al CPS, y los recursos del centro y/o comunitarios que pueden ofrecérsele al paciente, $\mathrm{o}$ en relación con el mismo.

\section{Acta del CPS}

Los asistentes al CPS disponen, en cada reunión semanal de 60 minutos, de una hoja de los casos que se van a presentar (fig. 2). Estos casos han sido previamente entregados a la administrativa del área de consultas externas, presente el día de la reunión. Así, la administrativa del CPS dispondrá de una información de partida, especificada en el acta, entregada por la persona que presenta el caso, la cual guiará su presentación. En dicha acta figurará información médica y psicosocial. Una vez completada la información en el acta del día, con la decisión tomada por el CPS, esta será introducida en la historia clínica del paciente. Una vez valorado el paciente o el familiar, el caso será derivado a la unidad, servicio o profesional que precise, en carácter ordinario o preferente. Como derivación preferente entendemos aquel caso que debemos priorizar en las agendas asistenciales por presentar uno o varios de los criterios de alta 


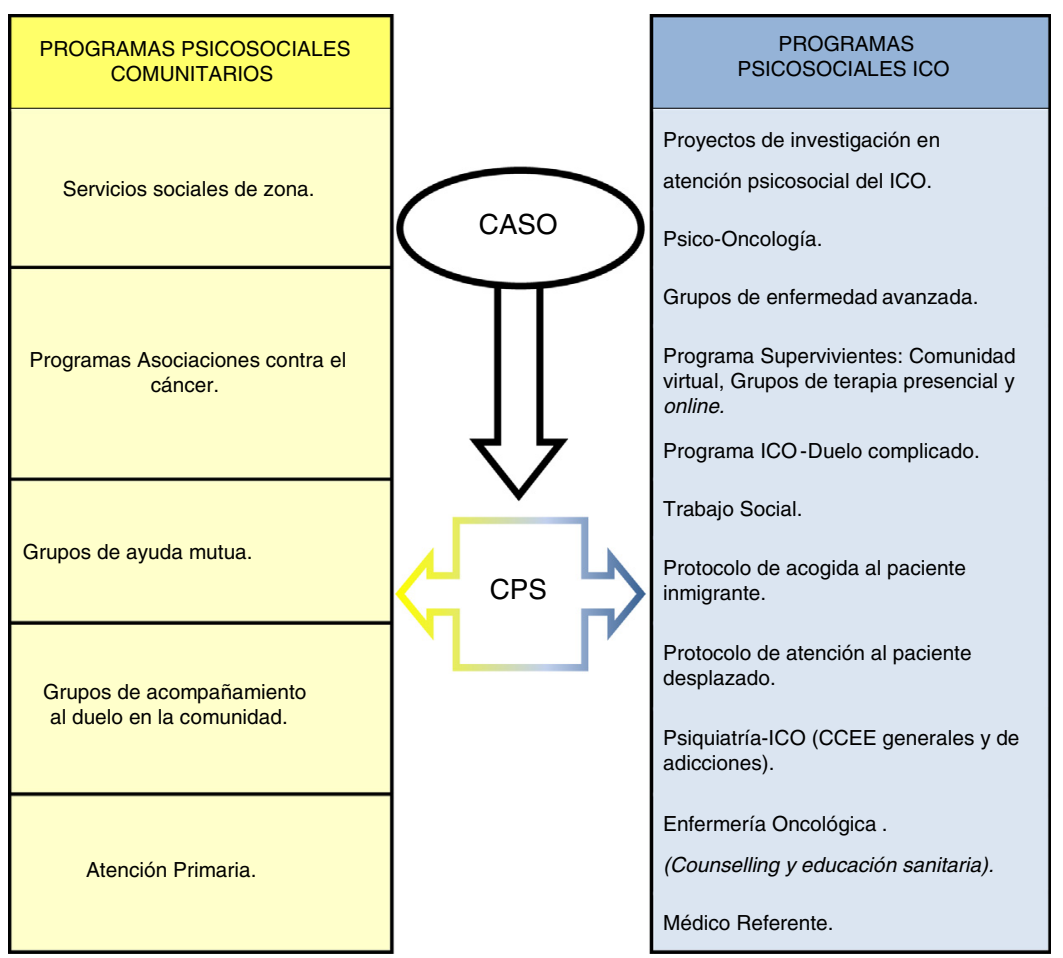

Figura 5. Programas y recursos de atención psicosocial del Comité Psicosocial (CPS).

complejidad o con altos niveles de malestar emocional, siendo visitado el paciente o el familiar en un periodo no superior a los 15 días.

\section{Agendas}

Los profesionales de los servicios de psicooncología, trabajo social y psiquiatría dispondrán de espacios reservados en sus agendas asistenciales para los casos derivados por el CPS. El tiempo de espera de una visita derivada desde el CPS no deberá superar las dos semanas desde la reunión.

Los criterios o indicadores de calidad del CPS recogidos en las actas del comité (fig. 2) y que hemos evaluado desde enero de 2017 a julio de 2018 han sido los siguientes: a) número de pacientes evaluados por el grado de complejidad y recursos asistenciales ofrecidos al paciente y/o familiar; b) número de casos que han sido priorizados y que han requerido atención psicosocial multidisciplinar rápida y frecuente (programación de la visita antes de dos semanas); c) número de actas elaboradas en el CPS por cada caso presentado; d) número de actas colgadas y firmadas en la historia clínica del paciente; y e) evaluación anual de la mejora en el bienestar emocional y la reducción de la complejidad psicosocial de los pacientes y familiares, una vez revisados en el CPS, un mes después de haber sido presentados.

$\mathrm{Al}$ no ser un estudio sobre seres humanos, sino de registro de casos, no ha sido necesaria la aprobación expresa del comité de ética.

\section{Análisis de los datos}

Los datos analizados corresponden al período entre el mes de enero de 2017 y el mes de junio de 2018. Para los análisis estadísticos se utilizó la versión 21.0 de IBM SPSS. Análisis de frecuencias y estadísticos descriptivos se utilizaron para la descripción de la muestra. Para comparar los valores en la escala visual analógica de malestar emocional, y dado que los datos no seguían una distribución normal, se llevó a cabo la prueba no paramétrica de Wilcoxon con rangos entre los valores pre y post comité. Entre estos, también se calcularon las frecuencias en los tres niveles de complejidad (baja-media-alta) antes y después de la atención psicosocial, a fin de comprobar variaciones entre pre y post comité.

\section{Resultados}

\section{Muestra}

Los resultados presentados corresponden a los criterios o indicadores de calidad alcanzados por el CPS señalados en el apartado anterior. Durante el período comprendido entre enero de 2017 y junio de 2018, el número de pacientes evaluados en el CPS ha sido de 144. De estos, 27 han requerido atención psicosocial prioritaria, siendo visitados en un periodo no superior a dos semanas. Las características sociodemográficas de estos pacientes se describen en la tabla 1. El número de actas elaboradas y firmadas por paciente en el comité asciende a 144, y han sido colgadas dentro de la historia clínica del paciente.

\section{Efectos de la revisión de casos del CPS}

Los pacientes discutidos por parte de los miembros del CPS fueron revisados en el mismo un mes después de su primera presentación. El malestar emocional de los pacientes después de haber sido visitados por el especialista acordado en el CPS (psicooncólogo, psiquiatra o trabajador social) muestra un descenso significativo con respecto a los valores registrados previamente $(p=0,001)$. En concreto, los pacientes reportaban una media inicial de 8,0 puntos en malestar emocional y un descenso a una media de 5,81 puntos después de haber sido considerados y derivados por el CPS al especialista del comité. El descenso se vio también reflejado en los niveles de complejidad. Así, antes de ser revisados por el CPS, el $70,4 \%$ de pacientes mostraban un alto nivel de complejidad psicosocial, el $22,2 \%$ mostraban un nivel medio y el 7,4\% mostraban un nivel bajo. Después de ser revisados y atendidos según las decisiones tomadas por el CPS, los porcentajes de pacientes en los 
Tabla 1

Características sociodemográficas de los pacientes analizados $(\mathrm{n}=27)$

\begin{tabular}{ll}
\hline Edad & \\
Media & 57,35 \\
Desviación típica & 12,56 \\
Mín.-máx. & $36-82$ \\
Estado civil, $n$ (\%) & \\
Soltero/a & $3(11,1)$ \\
Casado/a o con pareja & $12(59,3)$ \\
Viudo/a & $3(11,1)$ \\
Separado/a & $5(18,5)$ \\
Número de hijos, $n$ (\%) & \\
0 & $4(14,8)$ \\
1 & $7(25,9)$ \\
2 & $14(51,9)$ \\
3 & $2(7,4)$ \\
Situación laboral, $n$ (\%) & \\
Paro & \\
Incapacidad laboral temporal & $3(11,1)$ \\
Activo & $4(14,8)$ \\
Jubilado & $3(11,1)$ \\
No informa & $7(25,9)$ \\
& $10(37,0)$
\end{tabular}

distintos niveles de complejidad psicosocial mejoraron, reduciéndose al $48,1 \%$ en los enfermos con alta complejidad y aumentando hasta el 22,2\% los pacientes con baja complejidad psicosocial. El porcentaje de pacientes con niveles de complejidad media se mantuvo en valores similares (29,6\%).

\section{Discusión}

Los resultados y la información que proporciona el presente estudio dan respuesta a la ya conocida necesidad de abordar las dificultades del paciente oncológico desde un punto de vista multidisciplinar. Se trata de organizar la atención oncológica desde cada una de sus partes, asegurando la comunicación y la coordinación lógica y eficaz entre los profesionales de cada disciplina ${ }^{17}$. Esta atención multidisciplinar es especialmente relevante en cáncer, dado que se trata de una patología que requiere abordajes específicos a lo largo de su evolución, así como de una gran cantidad de recursos humanos y tecnológicos que están en constante cambio y renovación. Tal y como señalan Borràs y $\operatorname{Prades}^{18}$, la buena o mala gestión de los casos de cáncer puede tener considerables repercusiones, dada la complejidad clínica y asistencial de esta enfermedad. Así, estos autores recomiendan la cada vez más importante distribución multidisciplinar de las responsabilidades en los casos de cáncer, por su elevada incidencia, la distribución constante de competencias entre profesionales y la innovación terapéutica, así como su multimodalidad ${ }^{18}$.

Dentro de esta atención disciplinar en cáncer, abordar los aspectos psicosociales no solo ayuda a mejorar la calidad de vida de los pacientes, sino también a tomar una decisión del tratamiento que debe seguir un paciente en una unidad multidisciplinar de cáncer ${ }^{19}$, conocida en este caso como «unidad funcional de cáncer» en el Instituto Catalán de Oncología. Este modelo de CPS es pionero en la atención integral del enfermo y de su familia; sin embargo, podría ser comparado con las sesiones de objetivos que se realizan por parte del equipo asistencial en pacientes ingresados. Al no disponer de estudios previos que cuantifiquen la reducción del malestar emocional y de la complejidad psicosocial en pacientes presentados en estas sesiones de objetivos, centramos la atención sobre la mejora observada en malestar emocional y complejidad psicosocial de los casos presentados en el CPS. En concreto, observamos una mejora en el malestar emocional y en el nivel de complejidad psicosocial de los pacientes derivados al equipo de salud mental de oncología (psicooncólogos, psiquiatras y trabajadores sociales) desde el CPS. Esta mejora se traduce en una reducción de 2,19 puntos en malestar emocional, así como una disminución del 22\% de casos de alta complejidad psicosocial. Los resultados indican, entonces, que el CPS ofrece a los profesionales del centro un instrumento para poder derivar los casos de mayor complejidad que requieren atención preferente, pudiéndose beneficiar el paciente o familiar de una atención multidisciplinar de profesionales del área de la salud en general, y específicamente del área de la salud mental. Asimismo, desde un punto de vista de política o gestión sanitaria, el comité permite optimizar recursos, ofreciendo una alta eficacia en la resolución de casos complejos a nivel psicosocial.

Otras instituciones han publicado otros modelos de atención psicosocial, aunque pocos se han basado en los niveles de complejidad, poniendo hincapié en la comunicación médico-paciente ${ }^{1}$, o centrándose en población muy concreta, como pacientes de cáncer infantil oncológicos ${ }^{20}$. Por contra, ya el mismo Observatorio Qualy del Instituto Catalán de Oncología definió cuáles eran los criterios de complejidad de enfermos avanzados y terminales, y a partir de ellos propusieron las intervenciones y los seguimientos de equipos específicos en cuidados paliativos. Estas intervenciones estaban basadas en niveles de complejidad y de necesidades del enfermo ${ }^{21}$. De igual manera, desde el CPS se valora el nivel de complejidad y se decide el tipo de atención psicosocial que requiere el paciente $o$ familiar (psicooncología, psiquiatría o trabajo social). Otra aportación al estudio de la complejidad es la elaboración del ICD-Pal ${ }^{22}$, un instrumento diagnóstico de la complejidad en cuidados paliativos. A nivel psicosocial, ICD-Pal considera de compleja o de alta complejidad las siguientes situaciones: paciente con riesgo de suicidio, paciente que solicita adelantar el proceso de la muerte, paciente que presenta angustia existencial y/o sufrimiento espiritual, conflicto en la comunicación entre paciente y familia, conflicto en la comunicación entre paciente y equipo sanitario, y paciente que presenta afrontamiento emocional desadaptativo ${ }^{23}$. Algunas de estas situaciones o criterios son los mismos o semejantes a los utilizados en el presente modelo de CPS para definir el caso que se presenta en el comité como de alta complejidad: dificultades en el control de síntomas, ausencia del cuidador, paciente con miembros vulnerables a su cargo, ansiedad severa, depresión severa, ideación autolítica, desesperanza, y pérdida del sentido y del propósito de vida. Estos criterios surgen de un estudio Delphi realizado desde el Observatorio de Atención Psicosocial del Cáncer del Instituto Catalán de Oncología, pendiente de ser publicado.

Aunque el presente estudio muestra un modelo eficaz de atención psicosocial en cáncer, no está libre de limitaciones. El tamaño de la muestra, así como el hecho de que pertenezca a un solo centro sanitario (Hospital Duran i Reynals, Instituto Catalán de Oncología), limitan la externalización de los resultados. Sería necesario realizar más estudios con el objetivo de poder replicar los resultados logrados en otros centros, en relación con el beneficio a nivel psicosocial que el comité aporta.

\section{Financiación}

Este trabajo se ha realizado sin financiación.

\section{Conflicto de intereses}

Los autores declaran no tener ningún conflicto de intereses.

\section{Agradecimientos}

A los miembros del Comité Psicosocial del Hospital Duran i Rey-nals, Instituto Catalán de Oncología, sin los cuales no solo esteestudio no podría haberse realizado, sino que además no sería posi-ble el funcionamiento de dicho comité. 
A los miembros del Plan Director de Oncología de Catalũna y alInstituto Catalán de Oncología por su asesoramiento en la elabora-ción del presente estudio.

A Raquel Alcalde Cambronero por su apoyo como administrativa del Comité Psicosocial del Hospital Duran i Reynals, InstitutoCatalán de Oncología.

\section{Bibliografía}

1. Nancy E, Adler AE. Cancer Care for the Whole Patient: Meeting Psychosocial Health Needs. 1 st ed. Washington (DC): National Academies Press; 2008.

2. Henselmans I, Helgeson VS, Seltman H, de Vries J, Sanderman R, Ranchor AV. Identification and prediction of distress trajectories in the first year after a breast cancer diagnosis. Heal Psychol. 2010;29:160-8

3. Gil FL, Costa G, Pérez FJ, Salamero M, Sánchez N, Sirgo A. Adaptación psicológica y prevalencia de trastornos mentales en pacientes con cáncer. Med Clin. 2008;130:90-2.

4. Gil FL, Costa G, Pérez FJ. Does chemotherapy reduce stress? Palliat Support Care. 2010;8:455-60.

5. Gil FL, Costa G, Hilker I, Benito L. First anxiety, afterwards depression: Psychological distress in cancer patients at diagnosis and after medical treatment. Stress Heal. 2012;28:362-7.

6. Cook SA, Salmon P, Hayes G, Byrne A, Fisher PL. Predictors of emotional distress a year or more after diagnosis of cancer: A systematic review of the literature. Psychooncology. 2018;27:791-801.

7. Carlson LE, Bultz BD. Efficacy and medical cost offset of psychosocial interventions in cancer care: Making the case for economic analyses. Psychooncology. 2004; 13:837-49.

8. Travado L, Dalmas M. Psychosocial oncology care. En: Albreht T, Martín-Moreno JM, Jelenc M, Gorgojo L, Harris M, editores. European Guide for the Quality National Cancer Control Programmes. Ljubljana: National Institute of Public Health; 2015. p. 36-41.

9. Travado L, Reis JC, Watson M, Borràs JM. Psychosocial oncology care resources in Europe: A study under the European Partnership for Action Against Cancer (EPAAC). Psychooncology. 2017;26:523-30.

10. Borràs JM, Federation of Spanish Oncology Societies, Spanish Society of Pathology, Spanish Society of Palliative Care, Spanish Society of Oncology Nursing, Spanish Society of Haematology and Haemotherapy, et al. Institutional declaration in favour of the development of interdisciplinary cancer care in Spain. Clin Transl Oncol. 2010;12:587-9.

11. Borràs JM, Albreht T, Audisio R, Briers E, Casali P, Esperou H, et al., European Partnership Action Against Cancer consensus grou. Policy statement on multidisciplinary cancer care. Eur J Cancer. 2014;50:475-80.
12. Prades J, Borràs JM, Brechot JM, Faithfull S, Trama A, Casali PG. More than a European sound box: Capitalising on the added value of the European perspective in cancer treatment. En: Martín-Moreno JM, Albreht T, Rados Krnel S, editores. Boosting Innovation and Cooperation in European Cancer Control. Key Findings from the European Partnership for Action Against Cancer. Ljubljana: Nationa Institute of Public Health of the Republic of Slovenia and World Health Organization on behalf of the European Observatory on Health Systems and Policies; 2013. p. 117-40.

13. Galán M, Farran L, Aliste L, Hormigo G, Aranda H, Bettonica C, et al Multidisciplinary cancer care may impact on the postoperative mortality and survival of patients with oesophageal and oesophagogastric junction cancer: A retrospective cohort study. Clin Transl Oncol. 2015;17: 247-56.

14. Canadian Association of Psychosocial Oncology. Standards of psychosocial health services for persons with cancer and their families, 2010 [consultado 24 Oct 2018]. Disponible en: https://capo.ca/CAPOstandards.pdf.

15. Turner J, Zapart S, Pedersen K, Rankin N, Luxford K, Fletcher J. Clinical practice guidelines for the psychosocial care of adults with cancer. Psychooncology. 2005; $14: 159-73$

16. Gil F, Novellas A, Barbero E, Hollenstein MF, Maté J. Modelo de atención psicosocial en oncología: Standards. Psicooncologia. 2004;1:179-84.

17. Haward R. Organising a comprehensive framework for cancer control. En: Coleman MP, Alexe DM, Albreht T, McKee M, editores. Responding to the Challenge of Cancer Care in Europe. Ljubljana: Institute of Public Health of the Republic of Slovenia and European Observatory on Health Systems and Policies; 2008. p. 113-34.

18. Borràs JM, Prades J. El desarrollo de la atención multidisciplinar en la atención al cáncer. Encuentros Multidisciplinares. 2017;55:1-6.

19. Grassi L, Spiegel D, Riba M. Advancing psychosocial care in cancer patients. F1000Res. 2017;6:2083.

20. Masera G, Spinetta JJ, Jankovic M, Ablin AR, d'Angio GJ, van Dongen-Melman J, et al. Guidelines for assistance to terminally ill children with cancer: A report of the SIOP Working Committee on psychosocial issues in pediatric oncology. Med Pediatr Onco. 1999;32:44-8.

21. Gómez-Batiste X, Blay C, Martínez-Muñoz M, Lasmarías C, Vila L, Espinosa J, et al. The Catalonia WHO Demonstration Project of Palliative Care: Results at 25 years (1990-2015). J Pain Sympt Manage. 2016;52:92-9.

22. Martín-Roselló ML, Fernández-López A, Sanz-Amores R, Gómez-García R, Vidal-Españ F, Cia-Ramos R. IDC-PAL: instrumento diagnóstico de la complejidad en cuidados paliativos: documento de apoyo al PAI cuidados paliativos. Sevilla: Consejería de Igualdad, Salud y Política Sociales: 2014.

23. Soukup T, Lamb BW, Sarkar S, Arora S, Shah S, Darzi A, et al. Predictors of treatment decisions in multidisciplinary oncology meetings: A quantitative observational study. Ann Surg Oncol. 2016;23:4410-7. 